\title{
Efecto de la sustitución de grasas en salchichas de tilapia roja (Oreochromis sp.) por una mezcla de piel de cerdo y fibra de quinua
}

\section{Effect of fat replacement in red tilapia sausages (Oreochromis sp.) with a mixture of pork skin and quinoa fiber}

\author{
José Igor Hleap-Zapata'; Gloria Rodríguez-de-la-Pava²; Saúl Dussan-Sarria ${ }^{3}$
}

\begin{abstract}
${ }^{1}$ Ing. Pesquero, Ph.D. Universidad Nacional de Colombia, Facultad de Ingeniería y Administración. Palmira - Valle del Cauca, Colombia; e-mail: jihleapz@unal. edu.co; (Dhttps://orcid.org/0000-0001-9692-5443

${ }^{2}$ Ing. Química, M.Sc. Universidad de San Buenaventura. Cali - Valle del Cauca, Colombia; e-mail: gcrodrig@usbcali.edu.co; Dhttps://orcid.org/0000-00015403-4976
\end{abstract}

${ }_{3}^{3}$ Ing. Agrícola, Ph.D. Universidad Nacional de Colombia, Facultad de Ingeniería y Administración. Palmira - Valle del Cauca, Colombia; e-mail: sdussan@unal. edu.co; (Dhttps://orcid.org/0000-0001-9297-0781

Cómo citar: Hleap-Zapata, J.I.; Rodríguez-de-la-Pava, G.; Dussan-Sarria, S. 2020. Efecto de la sustitución de grasas en salchichas de tilapia roja (Oreochromis sp) por una mezcla de piel de cerdo y fibra de quinua. Rev. U.D.C.A Act. \& Div. Cient. 23(1):e1149. http://doi. org/10.31910/rudca.v23.n1.2020.1149

Artículo de acceso abierto publicado por Revista U.D.C.A Actualidad \& Divulgación Científica, bajo una licencia Creative Commons CC BY-NC 4.0

Publicación oficial de la Universidad de Ciencias Aplicadas y Ambientales U.D.C.A, Institución de educación superior Acreditada de Alta Calidad por el Ministerio de Educación Nacional.

Recibido: Enero 21 de 2019

Aceptado: Febrero 6 de 2020

Editado por: Ingeborg Zenner de Polanía

\section{RESUMEN}

La disminución del consumo de grasas saturadas aportadas por los alimentos de origen animal y la búsqueda de alimentos más sanos es una tendencia, a nivel mundial. La producción de tilapia en Colombia ha venido en franco crecimiento en las últimas décadas, lo cual, plantea la necesidad de búsqueda de alternativas para su procesamiento. Se evaluó la sustitución de grasas por una mezcla de piel de cerdo, agua y fibra de quinua (PCFQ) en salchichas de filete de tilapia. Se determinó la composición química de las salchichas elaboradas, el contenido calórico, el pH, las coordenadas de color CIELab, las pérdidas de humedad por cocción, la estabilidad de la emulsión, el perfil de textura y se estableció la apreciación sensorial. La adición de PCFQ conllevó a un aumento en la humedad y en las proteínas totales, gracias a la capacidad de retención de agua de la fibra de quinua. La salchicha con mayor adición de PCFQ (20\%) obtuvo un 48,2\% menos de grasas, un 32,3\% menos de calorías y un $31,1 \%$ de pérdida de humedad por cocción, con relación a la salchicha control. Se obtuvieron emulsiones cárnicas más estables, con buenos parámetros de perfil de textura - TPA. Con relación a los parámetros sensoriales, no se apreciaron diferencias significativas entre las salchichas elaboradas y la salchicha control. Por lo anterior, se concluye que, la mezcla, se puede usar como sustituto de grasas en la elaboración de salchichas de filete de tilapia, sin afectar sus propiedades fisicoquímicas y sensoriales.

Palabras clave: industria pesquera; piel de cerdo; quinua; salchichas de tilapia; grasas saturadas. 


\section{ABSTRACT}

The decrease in the consumption of saturated fats provided by animal foods and the search for healthier foods is a worldwide trend. Tilapia production in Colombia has been growing rapidly in recent decades, which raises the need to find alternatives for processing. The substitution of fats for a mixture of pork skin, water and quinoa fiber (PCFQ) in tilapia fillet sausages was evaluated. The chemical composition of the processed sausages, the caloric content, the $\mathrm{pH}$, the CIELab color coordinates, the cooking moisture losses, the emulsion stability, the texture profile and the sensory assessment were determined. The addition of PCFQ led to an increase in moisture and total proteins, thanks to the water retention capacity of quinoa fiber. The sausage with the highest PCFQ addition (20\%), obtained $48.2 \%$ less fat, $32.3 \%$ less calories and $31.1 \%$ loss of moisture from cooking compared to the control sausage. More stable meat emulsions were obtained with good texture profile parameters -TPA. Regarding sensory parameters, no significant differences were observed between processed sausages and control sausage. Therefore, it is concluded that the mixture can be used as a fat substitute in the preparation of tilapia fillet sausages, without affecting its physicochemical and sensory properties.

Keywords: fishing industry; pork skin; quinoa; tilapia sausages; saturated fat.

\section{INTRODUCCIÓN}

A nivel mundial y en Colombia, en particular, la producción acuícola de especies ícticas, se ha incrementado en las últimas décadas (FAO, 2015a). Entre las especies más comerciales, se encuentran la tilapia roja (Oreochromis sp.) y la tilapia nilótica (Oreochromis niloticus), con un 58,5\% del total de la producción en Colombia (AUNAP, 2013). Básicamente, esta producción se destina a la exportación, con algún porcentaje para el consumo nacional, a través de la venta entera, eviscerada o no, o como filetes congelados y una pequeña parte, para la transformación en productos procesados, tales como hamburguesas, filetes apanados, salchichas y nuggets.

Según datos aportados por FAO (2015b) y Shahidi \& Ambigaipalan (2015), los alimentos de origen hidrobiológico son de alta importancia para la seguridad alimentaria mundial. Debido a su naturaleza perecedera y a su gran demanda, una parte importante de estos recursos se procesa de diversas maneras (Pal \& Suresh, 2016). La tendencia mundial, de consumo de alimentos, presenta un incremento en aquellos con mayor aporte proteico, caso del pescado (Hajkowicz et al. 2012) y dentro de estos, se tienen, además del consumo en fresco, refrigerado o congelado, algunos alimentos procesados, tales como las salchichas tipo Frankfurt que, en Colombia, han ido adquiriendo mayor aceptabilidad. Sin embargo, el aporte calórico y el contenido de grasas adicionadas, generalmente, en altos porcentajes, necesarias para lograr una buena emulsificación de la masa muscular, reducen su aceptabilidad. Otros autores, como Hleap \& Rodríguez (2018), para salchichas elaboradas a partir de filetes de tilapia roja, obtuvieron un contenido de $2,64-2,70 \mathrm{~g} / \mathrm{kg}$ de lípidos; Granados-Conde et al. (2013), para salchichas fabricadas a partir de carne roja de atún, de 8,2g/kg de lípidos; Cabello et al. (2005), para salchichas hechas con pescado obtenido de la fauna acompañante de la captura de camarón, de $3,0 \mathrm{~g} / \mathrm{kg}$ de lípidos y Reddy et al. (2020), para salchichas adicionadas con aislados proteicos obtenidos del fileteado del Pangas (Pangasius hypophthalmus), de 5,25g/mg de lípidos. Se puede inferir que, aunque son valores bajos comparados con una salchicha comercial elaborada a partir de pescado $(7,4 \mathrm{~g} /$ $\mathrm{kg}$ ), adquirida en una tienda especializada en productos alimenticios de origen hidrobiológico de la ciudad de Cali, Colombia, siguen siendo altos. La tendencia mundial es la búsqueda de alimentos con menor contenido de grasas (Limberger et al. 2011), procurando que no superen el 30\% de las calorías totales en la ingesta diaria, con el fin de prevenir diferentes enfermedades y reducir los niveles de colesterol alto (Ferguson, 2010; McAfee et al. 2010), en consecuencia, es importante la producción de derivados cárnicos y pesqueros con niveles reducidos de grasa y colesterol y con un perfil adecuado de ácidos grasos (Pacheco-Pérez et al. 2011).

La importancia del uso de las grasas, se relaciona con las propiedades sensoriales, tales como el sabor, la ternura, la jugosidad, la apariencia y la textura, así como con la vida útil y la aceptabilidad de los productos cárnicos (Yilmaz, 2005; Intarasirisawat et al. 2014). Varias investigaciones han buscado sustitutos de las grasas en los productos cárnicos, de tal forma que no se afecten dichas características. Para esto, se ha recurrido al uso de aceites vegetales (Rueda-Lugo et al. 2006; Choi et al. 2010), geles del tejido conectivo (Osburn et al. 1997; Benelli et al. 2015) y fibras dietéticas y carbohidratos en general (Limberger et al. 2011; Candogan \& Kolsarici, 2003). La fibra de quinua (Chenopodium quinoa W.) posee diversas propiedades funcionales, que se pueden utilizar en la elaboración de múltiples alimentos de origen cárnico y pesquero (Miranda et al. 2012; Arzapalo et al. 2015). Al respecto, la adición de este tipo de fibras en productos cárnicos emulsionados conlleva a un mejoramiento en las características de calidad, tales como la textura y el rendimiento de cocción, al presentarse una alta capacidad de retención de humedad y una mejor unión del agua y la grasa (Tokusoglu \& Kemal, 2003; Serin \& Sayar, 2017). Por otro lado, la piel de cerdo en la industria procesadora de salchichas, se aplica desde hace mucho tiempo, gracias a la presencia de tejido conectivo, rico en colágeno (Osburn et al. 1997; Yang \& Shu, 2014; Benelli et al. 2015), el cual, en el proceso térmico propio de la elaboración de salchichas, se transforma en gelatina, aumentando la firmeza, la textura y la estabilidad de las mismas (Weston et al. 2002; Hsu \& Sun, 2006).

La mezcla de fibra de quinua y piel de cerdo aplicada a una salchicha, se presenta como una alternativa para disminuir la cantidad total de grasas, en este tipo de productos emulsionados. El objetivo de este estudio fue evaluar el efecto de la piel de cerdo mezclada con agua y fibra de quinua PCFQ, como sustituto parcial de la grasa, en las características de calidad de salchichas tipo Frankfurt, elaboradas a partir de filetes de tilapia roja (Oreochromis sp). 


\section{MATERIALES Y MÉTODOS}

Muestras de estudio. Las salchichas fueron elaboradas en el Laboratorio de Tecnología de Carnes de la Universidad Nacional de Colombia, sede Palmira, a partir de una formulación previamente desarrollada, validada y estandarizada (Hleap \& Velasco, 2010; Pinzón-Zárate et al. 2015). Se utilizaron filetes de tilapia ( $\mathrm{pH} \mathrm{6,6)}$ y grasa dorsal de cerdo, adquiridos en un supermercado de la ciudad de Palmira, departamento del Valle del Cauca, Colombia y se mantuvieron a $-3 \pm 1^{\circ} \mathrm{C}$, durante 24 horas, antes de su utilización, para lo cual, previamente fueron descongelados. La piel de cerdo, se adquirió directamente en un distribuidor de carnes de la misma ciudad y para su utilización, se troceó en tiras de, aproximadamente, $5 \mathrm{~cm}$ de largo, que se almacenaron a $-20 \pm 1^{\circ} \mathrm{C}$, hasta el momento de su uso. La fibra de quinua, se obtuvo por compra directa a una empresa de molinería de cereales, ubicada en la ciudad de Pasto, departamento de Nariño, Colombia. Se prepararon tres lotes de $6 \mathrm{~kg}$ cada uno, para cada tratamiento.

Se elaboró una mezcla homogénea conformada por piel de cerdo, agua y fibra de quinua (PCFQ), en proporción de 2:2:1, obteniendo como resultado una pasta de textura esponjosa. Para lograrlo, la piel de cerdo se sometió a cocción, a $82^{\circ} \mathrm{C}$, durante 45 minutos $\mathrm{y}$, posteriormente, se molió en un equipo Javar ${ }^{\circledR}$ M121 Mill Javar Bogotá, Colombia, con una rejilla de $3 \mathrm{~mm}$ de diámetro. Los tres ingredientes (el agua en forma de hielo), se homogenizaron en un cuter (Hobart 84181 D Corporation Troy, Ohio USA). Se elaboraron cuatro formulaciones de salchichas con diferentes concentraciones de PCFQ, que se aplicaron como sustituto parcial de la grasa y una formulación control sin adición de la mezcla PCFQ (Tabla 1). Para la elaboración de las salchichas, los filetes de tilapia se molieron durante 2,5 minutos, usando un cuter (Hobart 84181 D Corporation Troy, Ohio USA) y, posteriormente, se le agregaron la sal y los demás ingredientes, mezclando por tres minutos más. La mezcla PCFQ y la grasa dorsal de cerdo, previamente troceada, se añadieron y se emulsionaron durante dos minutos, para una homogenización adicional de cinco minutos. La temperatura de la emulsión, se mantuvo a $10 \pm 1^{\circ} \mathrm{C}$. Las salchichas, se embutieron en tripa artificial (Amicel $^{\circledR}$ Alico S.A., Medellín, Colombia), de $22 \mathrm{~mm}$ de diámetro y se amarraron, a una longitud de $12 \mathrm{~cm}$, correspondiendo a un peso aproximado de $60 \mathrm{~g}$. A continuación, se sometieron durante $15 \mathrm{minu}-$ tos a escaldado, a $80^{\circ} \mathrm{C}$ temperatura del agua y $72^{\circ} \mathrm{C}$ temperatura interna, medida con un termómetro de aguja (Chektemp HI 98051 HANNA Instruments Ltda., Leighton Buzzard Bedfordshire, England). Luego, se sometieron a un choque térmico en agua fría $\left(10^{\circ} \mathrm{C}\right)$, durante 15 minutos. Finalmente, se empacaron en bolsas plásticas, utilizando una máquina de sellado al vacío (EGARVAC S.C.P. Basic B Vacarisses, Barcelona, España) y se almacenaron a 4 $\pm 1^{\circ} \mathrm{C}$, durante tres días, antes de los análisis. Cada lote, se identificó con un código numérico diferente.

Composición proximal, contenido calórico, $\mathrm{pH}$ y medición de color. Se determinó la composición proximal de las salchichas utilizando los métodos estándar de la AOAC 2003. El porcentaje de humedad, se midió de acuerdo con el método 925.10, para lo cual, el porcentaje de humedad se estipuló comparando los pesos antes y después del secado en horno, a $105^{\circ} \mathrm{C}$, durante ocho horas, utilizando una balanza de humedad (PCE-MB 50, Ibérica S, L, España). El contenido de grasa, se determinó de acuerdo con el método Soxhlet (método 920.35), empleando un equipo SKZ1057B, SKZ Industrial Co., Limited Shandong, China y el contenido de proteína, se definió usando un analizador de nitrógeno Kjeldahl automático (K9840, Nanbei, Mainland, China), según el método 991.20. El contenido calórico, se determinó definiendo las calorías totales en Kcal/100, según el método de la bomba calorimétrica de Berthelot-Mahler, usando un calorímetro Parr 1261, Paff Co., Frankfurt, Alemania. Se determinó el pH de las salchichas antes y después del escaldado, empleando un medidor de $\mathrm{pH}$ tipo potenciómetro (modelo 340, Metter-Toledo, Schwerzenbach, Suiza); para esto, se mezclaron $5 \mathrm{~g}$ de la muestra con $20 \mathrm{ml}$ de agua destilada en un homogenizador,

Tabla 1. Formulaciones de las salchichas de filete de tilapia bajas en grasa, con diferentes niveles de mezcla PCFQ.

\begin{tabular}{|c|c|c|c|c|c|}
\hline \multirow{3}{*}{ Ingredientes } & \multicolumn{5}{|c|}{ Tratamiento (nivel de PCFQ*) } \\
\hline & \multirow{2}{*}{ Control } & $\mathbf{T}_{1}$ & $\mathbf{T}_{2}$ & $\mathbf{T}_{3}$ & $\mathbf{T}_{4}$ \\
\hline & & $8 \%$ & $12 \%$ & $16 \%$ & $20 \%$ \\
\hline Filetes de tilapia & 50 & 50 & 50 & 50 & 50 \\
\hline $\begin{array}{c}\text { Grasa dorsal de } \\
\text { cerdo }\end{array}$ & 30 & 22 & 18 & 14 & 10 \\
\hline PCFQ & 0 & 8 & 12 & 16 & 20 \\
\hline Hielo & 20 & 20 & 20 & 20 & 20 \\
\hline Total & 100 & 100 & 100 & 100 & 100 \\
\hline Sal & 1,5 & 1,5 & 1,5 & 1,5 & 1,5 \\
\hline Polifosfatos & 0,2 & 0,2 & 0,2 & 0,2 & 0,2 \\
\hline
\end{tabular}

*La proporción de la formulación de la mezcla de piel de cerdo y fibra de quinua fue piel de cerdo: agua: fibra de quinua = 2: 2: 1. 
durante un minuto. La determinación del color, se realizó utilizando un colorímetro (Kónica, Micolta Chroma Meter CR400, Japón), con iluminante D65 y observador de $2^{\circ}$ (equipo calibrado con una placa estándar con valores de referencia $Y=89,5, \mathrm{x}=0,3176$ y y $=$ 0,3340). Las mediciones, se expresaron en términos de luminosidad L* y de los parámetros de cromaticidad a* y b*. A partir de estos parámetros fueron calculadas las coordenadas $\mathrm{C}$ (índice de saturación) y h (tono), aplicando las ecuaciones 1 y 2 , respectivamente. Cada muestra se midió tres veces.

$$
\begin{aligned}
& \mathrm{C}=\left(\mathrm{a}^{* 2}+\mathrm{b}^{* 2}\right)^{1 / 2} \\
& \mathrm{~h}=\arctan \left(\mathrm{b}^{*} / \mathrm{a}^{*}\right)
\end{aligned}
$$

Ecuación 1

Ecuación 2

Pérdida de humedad por cocción y estabilidad de la emulsión. La pérdida de humedad durante el escaldado para las diferentes muestras, se midieron de acuerdo con la metodología propuesta por Choe et al. (2013), mediante el cálculo de las diferencias de peso antes y después del escaldado $\left(80^{\circ} \mathrm{C}, 15\right.$ minutos). Después de enfriar las salchichas durante 30 minutos, se pesaron y se calculó el porcentaje de pérdida de peso, utilizando la ecuación 3.

Pérdida de cocción $=\frac{\mathrm{P} 1-\mathrm{P} 2}{\mathrm{p} 1} \times 100$

Ecuación 3

Donde, $\mathrm{P}_{1}$ - Peso de la salchicha antes del escaldado y $\mathrm{P}_{2}-$ Peso de la salchicha después del escaldado.

Aplicando la metodología propuesta por Ensor et al. (1987) y las modificaciones propuestas por Choe et al. (2013), se analizó la estabilidad de las emulsiones elaboradas. Para los cálculos finales, se utilizaron las ecuaciones 4 y 5 .

$$
\begin{gathered}
\text { Grasa liberada }=\left[\frac{\text { Capa de grasa }(\mathrm{ml})}{\text { peso de la masa de la carne cruda }(\mathrm{g})}\right] \times 100 \\
\text { Ecuación } 4
\end{gathered}
$$$$
\text { Agua liberada }=\left[\frac{\text { Capa de agua }(\mathrm{ml})}{\text { peso de la masa de la carne cruda }(\mathrm{g})}\right] \times 100
$$

Ecuación 5

Análisis de perfil de textura. Las salchichas, se sometieron a la prueba de perfil de textura, la cual, se realizó en tres réplicas para cada muestra. Para esto, las salchichas, después del escaldado, se enfriaron a temperatura ambiente durante 45 minutos y se midieron las propiedades de textura, utilizando un texturómetro (Shimadzu Universal Tester EZTest EZ-S, Shimadzu Europa GmbH). Las condiciones de prueba fueron las siguientes: trazo $20 \mathrm{x}$ g, velocidad de prueba 2,0mm/s, distancia 20,0mm. Se determinó dureza $(\mathrm{N})$, elasticidad, cohesión, gomosidad $(\mathrm{N})$ y masticabilidad $(\mathrm{N})$.

Evaluación sensorial. La evaluación sensorial, la selección y el entrenamiento de los panelistas, se llevó a cabo en las instalaciones de la Universidad de San Buenaventura, Cali. Se trabajó con 15 panelistas entrenados en productos cárnicos procesados, con salchichas comerciales. Se llevó a cabo durante cuatro semanas, tiempo que permitió familiarizar a los panelistas con las características senso- riales de las salchichas elaboradas a partir de los filetes de tilapia. Para las degustaciones, las salchichas de cada una de las muestras, se calentaron en agua a $80^{\circ} \mathrm{C}$, durante 25 minutos; posteriormente, se enfriaron hasta $25^{\circ} \mathrm{C}$, se cortaron en trozos de $2 \mathrm{~cm}$ de largo y se suministraron a los panelistas. La evaluación sensorial, se realizó bajo iluminación fluorescente blanca y todos los parámetros sensoriales: color, sabor, ternura, jugosidad, sabor calentado y aceptabilidad general; se evaluaron utilizando una escala hedónica de siete puntos ( 1 = no me gusta y 7 = me gusta muchísimo).

Análisis estadístico. Todas las mediciones, se realizaron por triplicado y los valores se muestran como el promedio más la desviación estándar. El análisis de varianza, se aplicó en todos los parámetros evaluados, utilizando el paquete estadístico IBM SPSS Statics 19. Se obtuvo un análisis de varianza ANOVA y se aplicó la prueba Tukey, para determinar las diferencias estadísticas entre las muestras $(p=0,05)$.

\section{RESULTADOS Y DISCUSIÓN}

En la tabla 2, se aprecia la composición proximal, el valor de $\mathrm{pH}$ y los parámetros de color de la mezcla PCFQ. Para las salchichas elaboradas, los resultados obtenidos mostraron diferencias significativas $(\mathrm{p}<0,05)$, en cuanto a humedad y lípidos en todos los tratamientos (Tabla 3). A medida que aumentó la concentración de PCFQ, aumentó la humedad, mientras que la grasa disminuyó $(p<0,05)$, posiblemente, debido a la alta absorción que presenta la fibra de quinua hacia el agua, mientras que la aceptación de grasas es mínima. Diferentes investigaciones relacionan el uso de fibras vegetales, tales como las fibras de trigo, fibras de los residuos de makgeolli, fibras regeneradas de celulosa y fibras de konjac mezcladas con carragenano, como reemplazo de grasas en salchichas tipo Frankfurt (Choe et al. 2013; Choi et al. 2014; Zhao et al. 2018; Hemung \& Sriuttha, 2014), logrando disminuir dicho contenido en valores significativos. En la presente investigación, el uso de la mezcla PCFQ presentó, con relación a los parámetros físico-químicos, resultados similares a los hallados en las citadas investigaciones. Finalmente, el contenido de proteína, se incrementó con el aumento de la mezcla de PCFQ, mientras que las cenizas no se vieron afectadas $(\mathrm{p}<0,05)$.

El decremento calórico observado se atribuye, tanto al menor contenido graso en cada uno de los tratamientos aplicados como a la mayor concentración de la mezcla PCFQ adicionada en cada tratamiento. Para las salchichas control, se obtuvo un valor de $313,68 \mathrm{kcal} / 100 \mathrm{~g}$, mientras que las salchichas de los diferentes tratamientos variaron entre 212,27 y $301,36 \mathrm{kcal} / 100 \mathrm{~g}$, lo cual, significa una reducción de entre 3,92 y 32,32\%. A mayor concentración de PCFQ y menor adición de grasa en cada tratamiento, se observó un menor valor calórico, que concuerda con los resultados mostrados por Choe et al. (2013), en salchichas con adición de piel de cerdo, agua y fibra de cerdo.

El pH de las salchichas no cocidas presentó una variación de 6,28 a 6,35 , medida que aumentó con el incremento de la concentración de PCFQ, lo cual, se puede explicar por el mayor valor de $\mathrm{pH}$ en la mezcla PCFQ $(7,26)$. Por el contrario, en las salchichas cocinadas no 
Tabla 2. Composición química y valores de pH de la mezcla PCFQ.

\begin{tabular}{|c|c|c|}
\hline \multicolumn{2}{|c|}{ Parámetro } & Mezcla PCFQ* \\
\hline \multicolumn{2}{|c|}{ Humedad } & $62,47 \pm 1,17$ \\
\hline \multicolumn{2}{|c|}{ Proteína } & $10,25 \pm 0,68$ \\
\hline \multicolumn{2}{|c|}{ Lípidos } & $8,34 \pm 0,57$ \\
\hline \multicolumn{2}{|c|}{ Cenizas } & $0,33 \pm 0,04$ \\
\hline \multicolumn{2}{|c|}{$\mathrm{pH}$} & $7,26 \pm 0,02$ \\
\hline \multirow{3}{*}{ Color } & $\mathrm{L}^{*}$ & $80,02 \pm 1,54$ \\
\hline & $a^{*}$ & $0,38 \pm 0,28$ \\
\hline & $\mathrm{b}^{*}$ & $14,44 \pm 0,31$ \\
\hline
\end{tabular}

Tabla 3. Efecto de la mezcla PCFQ en los parámetros fisicoquímicos de las salchichas elaboradas.

\begin{tabular}{|c|c|c|c|c|c|}
\hline \multirow{2}{*}{ Parámetro } & \multicolumn{5}{|c|}{ Tratamiento (nivel de PCFQ*) } \\
\cline { 2 - 5 } & \multirow{2}{*}{ Control } & $\mathbf{T}_{\mathbf{1}}$ & $\mathbf{T}_{\mathbf{2}}$ & $\mathbf{T}_{\mathbf{3}}$ & $\mathbf{T}_{4}$ \\
\cline { 2 - 6 } & & $\mathbf{8} \%$ & $\mathbf{1 2} \%$ & $\mathbf{1 6 \%}$ & $\mathbf{2 0} \%$ \\
\hline Humedad & $60,28 \pm 0,58^{\mathrm{e}}$ & $64,07 \pm 0,72^{\mathrm{d}}$ & $66,98 \pm 0,58^{\mathrm{c}}$ & $67,76 \pm 0,42^{\mathrm{b}}$ & $69,12 \pm 0,47^{\mathrm{a}}$ \\
\hline Proteína & $12,23 \pm 0,08^{\mathrm{c}}$ & $12,91 \pm 0,11^{\mathrm{d}}$ & $13,65 \pm 0,06^{\mathrm{c}}$ & $13,88 \pm 0,42^{\mathrm{b}}$ & $14,25 \pm 0,41^{\mathrm{a}}$ \\
\hline Lípidos & $21,12 \pm 0,98^{\mathrm{a}}$ & $18,52 \pm 0,76^{\mathrm{b}}$ & $15,99 \pm 0,82^{\mathrm{c}}$ & $12,02 \pm 0,53^{\mathrm{d}}$ & $10,94 \pm 0,50^{\mathrm{e}}$ \\
\hline Cenizas & $1,84 \pm 0,03$ & $1,97 \pm 0,03$ & $1,92 \pm 0,04$ & $1,89 \pm 0,04$ & $1,85 \pm 0,03$ \\
\hline Valor calórico (kcal/100g) & $313,68 \pm 0,67^{\mathrm{a}}$ & $301,36 \pm 0,41^{\mathrm{b}}$ & $281,72 \pm 0,52^{\mathrm{c}}$ & $237,66 \pm 0,37^{\mathrm{d}}$ & $212,27 \pm 0,42^{\mathrm{e}}$ \\
\hline pH sin cocinar & $6,28 \pm 0,03^{\mathrm{c}}$ & $6,30 \pm 0,04^{\mathrm{b}}$ & $6,32 \pm 0,03^{\mathrm{b}}$ & $6,34 \pm 0,03^{\mathrm{a}}$ & $6,35 \pm 0,04^{\mathrm{a}}$ \\
\hline pH cocinadas & $6,42 \pm 0,02^{\mathrm{b}}$ & $6,45 \pm 0,03^{\mathrm{a}}$ & $6,49 \pm 0,02^{\mathrm{a}}$ & $6,51 \pm 0,02^{\mathrm{a}}$ & $6,53 \pm 0,02^{\mathrm{a}}$ \\
\hline Pérdida de humedad por cocción & $5,04 \pm 0,58^{\mathrm{a}}$ & $4,31 \pm 0,39^{\mathrm{b}}$ & $3,88 \pm 0,22^{\mathrm{c}}$ & $3,52 \pm 0,29^{\mathrm{c}}$ & $3,47 \pm 0,24^{\mathrm{c}}$ \\
\hline \multicolumn{7}{|c|}{ Estabilidad de la emulsión } & & \\
\hline Exudación de grasa (\%) & $0,39 \pm 0,03^{\mathrm{a}}$ & $0,31 \pm 0,02^{\mathrm{b}}$ & $0,22 \pm 0,02^{\mathrm{c}}$ & $0,18 \pm 0,01^{\mathrm{c}}$ & $0,15 \pm 0,01^{\mathrm{c}}$ \\
\hline Exudación de agua (\%) & $6,11 \pm 0,43^{\mathrm{a}}$ & $4,66 \pm 0,34^{\mathrm{b}}$ & $3,24 \pm 0,29^{\mathrm{b}}$ & $1,15 \pm 0,42^{\mathrm{b}}$ & $1,02 \pm 0,40^{\mathrm{b}}$ \\
\hline
\end{tabular}

Todos los valores son medios \pm desviación estándar de tres repeticiones.

${ }^{a-e}$ medias dentro de filas con diferentes letras son significativamente diferentes $(p<0,05)$.

*La proporción de la formulación de la mezcla de piel de cerdo y fibra de quinua fue piel de cerdo: agua: fibra de quinua = 2: 2: 1.

se presentó variación estadísticamente significativa en los diferentes tratamientos con adición de PCFQ, que concuerda con los trabajos presentados por Bessa et al. (2016) y Souza et al. (2017), en salchichas de tilapia adicionadas con inulina y salchichas tipo Frankfurt, adicionadas con colágeno hidratado, respectivamente, en donde no se presentaron diferencias significativas $(\mathrm{p}<0,05)$, en los valores de $\mathrm{pH}$.

Los parámetros de color de las muestras presentaron los mayores valores de $L^{*}$ para la salchicha control $(p<0,05)$, en los tratamientos crudos y cocidos (Tabla 4). Los valores de $L^{*}$ y a* variaron con el aumento de la concentración de PCFQ y con la disminución en las grasas. Lo anterior conllevó a un color significativamente más oscuro y más rosado en las salchichas crudas adicionadas con PCFQ. Zakaria \& Sarbon (2018) mostraron datos similares, al adicionar hidrolizado de pescado en salchichas emulsionadas elaboradas a base de pescado. Por otro lado, se presentó un incremento no significativo en los valores de la coordenada $b^{*}$, tanto en las salchichas crudas como en las cocidas, con el aumento de la concentración de
PCFQ, debido, probablemente, a la coloración de la piel de cerdo, que es más amarilla en la mezcla utilizada, según anotaron en su trabajo Choe et al. (2013).

La capacidad de retención de agua en las salchichas está determinada por la pérdida de humedad por cocción a que son sometidas las mismas y esto, a su vez, depende de la capacidad de emulsión para inmovilizar la grasa y el agua, que repercute en la aceptación de las salchichas (Sorland et al. 2004). Se puede apreciar que, a medida que aumentó la concentración de PCFQ, también aumentó la pérdida de agua por cocción en las salchichas $(p<0,05)$, valor que fluctuó entre 4,31 y $3,47 \%$, siendo la de menor pérdida la correspondiente al 20\% de PCFQ, lo cual, indica que la mezcla utilizada posee una buena capacidad de retención de agua durante el tratamiento térmico (Tabla 3). Los datos obtenidos en esta investigación concuerdan con los presentados por Cheetangdee (2017), quien observó una pérdida de agua por cocción del 4\% en salchichas adicionadas con una mezcla de aceite de soya y proteína aislada de soya preemulsionada. 
Tabla 4. Efecto de la mezcla de piel de cerdo y fibra de quinua (PCFQ), en los parámetros de color y de textura en las salchichas elaboradas.

\begin{tabular}{|c|c|c|c|c|c|c|}
\hline \multirow{3}{*}{\multicolumn{2}{|c|}{$\begin{array}{l}\text { Parámetro } \\
\text { Control }\end{array}$}} & \multicolumn{5}{|c|}{ Tratamiento (nivel de PCFQ*) } \\
\hline & & \multirow{3}{*}{$\begin{array}{c}\mathbf{T}_{1} \\
\mathbf{8 \%} \\
75,24 \pm 0,68^{\mathrm{a}}\end{array}$} & \multirow{2}{*}{$\begin{array}{c}\mathrm{T}_{2} \\
12 \%\end{array}$} & \multirow{2}{*}{$\begin{array}{c}T_{3} \\
16 \%\end{array}$} & \multirow{2}{*}{$\begin{array}{c}\mathrm{T}_{4} \\
20 \%\end{array}$} & \\
\hline & & & & & & \\
\hline \multirow{5}{*}{ Sin cocinar } & $\mathrm{L}^{*}$ & & $72,43 \pm 0,29^{a}$ & $70,18 \pm 0,34^{a}$ & $68,12 \pm 0,68^{\mathrm{a}}$ & $66,28 \pm 0,47^{\mathrm{a}}$ \\
\hline & $a^{*}$ & $4,31 \pm 0,18^{\mathrm{a}}$ & $4,41 \pm 0,15^{\mathrm{a}}$ & $4,32 \pm 0,17^{\mathrm{a}}$ & $4,34 \pm 0,15^{\mathrm{a}}$ & $4,36 \pm 0,16^{\mathrm{a}}$ \\
\hline & $\mathrm{b}^{*}$ & $16,54 \pm 0,31^{\mathrm{b}}$ & $16,89 \pm 0,33^{\mathrm{ab}}$ & $17,03 \pm 0,39^{\mathrm{ab}}$ & $17,52 \pm 0,32^{\mathrm{a}}$ & $17,97 \pm 0,35^{\mathrm{a}}$ \\
\hline & $\mathrm{C}$ & $17,09 \pm 0,28^{a}$ & $17,46 \pm 0,36^{a}$ & $17,57 \pm 0,25^{a}$ & $18,05 \pm 0,33^{a}$ & $18,49 \pm 0,38^{a}$ \\
\hline & $\mathrm{h}$ & $75,39 \pm 041^{\mathrm{b}}$ & $75,37 \pm 0,35^{\mathrm{b}}$ & $75,77 \pm 0,42^{b}$ & $76,08 \pm 0,41^{\mathrm{b}}$ & $76,36 \pm 0,40^{\mathrm{b}}$ \\
\hline \multirow{5}{*}{ Cocinadas } & $\mathrm{L}^{*}$ & $73,04 \pm 0,84^{\mathrm{a}}$ & $71,76 \pm 0,88^{\mathrm{a}}$ & $69,15 \pm 0,73^{\mathrm{b}}$ & $67,06 \pm 0,75^{\mathrm{b}}$ & $65,78 \pm 0,72^{\mathrm{b}}$ \\
\hline & $a^{*}$ & $4,28 \pm 0,27$ & $4,30 \pm 0,29$ & $4,34 \pm 0,25$ & $4,36 \pm 0,41$ & $4,37 \pm 0,39$ \\
\hline & $b^{*}$ & $11,67 \pm 0,38^{\mathrm{b}}$ & $11,98 \pm 0,42^{\mathrm{ab}}$ & $12,14 \pm 0,40^{\mathrm{a}}$ & $12,65 \pm 0,45^{\mathrm{a}}$ & $12,81 \pm 0,48^{2}$ \\
\hline & $\mathrm{C}$ & $12,43 \pm 0,21^{\mathrm{a}}$ & $12,73 \pm 0,19^{\mathrm{a}}$ & $12,89 \pm 0,23^{\mathrm{a}}$ & $13,38 \pm 0,18^{a}$ & $13,53 \pm 0,18^{a}$ \\
\hline & $\mathrm{h}$ & $69,85 \pm 0,52^{\mathrm{b}}$ & $70,25 \pm 0,54^{\mathrm{b}}$ & $70,32 \pm 0,59^{\mathrm{b}}$ & $70,98 \pm 0,50^{\mathrm{b}}$ & $71,16 \pm 0,52^{b}$ \\
\hline \multirow{5}{*}{ TPA } & Dureza $(\mathrm{N})$ & $46,77 \pm 0,72^{c}$ & $60,63 \pm 0,25^{b}$ & $77,49 \pm 0,61^{\mathrm{a}}$ & $83,75 \pm 0,59^{\mathrm{a}}$ & $91,85 \pm 0,66^{a}$ \\
\hline & Elasticidad & $0,96 \pm 0,01^{a}$ & $0,85 \pm 0,07^{\mathrm{b}}$ & $0,83 \pm 0,06^{\mathrm{b}}$ & $0,82 \pm 0,05^{\mathrm{b}}$ & $0,80 \pm 0,05^{\mathrm{b}}$ \\
\hline & Cohesividad & $0,31 \pm 0,02^{c}$ & $0,32 \pm 0,04^{\mathrm{bc}}$ & $0,33 \pm 0,03^{\mathrm{ab}}$ & $0,34 \pm 0,02^{a}$ & $0,35 \pm 0,03^{\mathrm{a}}$ \\
\hline & Gomosidad (N) & $12,87 \pm 0,31^{\mathrm{c}}$ & $18,98 \pm 0,28^{\mathrm{b}}$ & $26,03 \pm 0,31^{\mathrm{a}}$ & $29,44 \pm 0,29^{a}$ & $31,89 \pm 0,33^{a}$ \\
\hline & Masticabilidad (N.mm) & $11,65 \pm 0,15^{\mathrm{b}}$ & $13,79 \pm 0,21^{\mathrm{b}}$ & $19,18 \pm 0,27^{a}$ & $20,05 \pm 0,22^{a}$ & $22,42 \pm 0,26^{a}$ \\
\hline
\end{tabular}

$L^{*}: 0=$ negro y $100=$ blanco; $a^{*}:-60=$ verde y $+60=$ rojo; $b^{*}:-60=$ azul y $+60=$ amarillo; $\mathrm{h}-$ tono: $90^{\circ}=$ amarillo, $180^{\circ}=$ verde y $0^{\circ}$

= rojo; $\mathrm{C}-$ índice de saturación: distancia desde las coordenadas en el origen hasta el punto de color determinado.

Todos los valores son medios \pm desviación estándar de tres repeticiones.

${ }^{a-c}$ medias dentro de filas con diferentes letras son significativamente diferentes $(p<0,05)$.

*La proporción de la formulación de la mezcla de piel de cerdo y fibra de quinua fue piel de cerdo: agua: fibra de quinua = 2:2:1.

Igualmente, coinciden con Choe et al. (2013), quienes presentaron valores de pérdida de agua por cocción, que variaron entre $6,43 \mathrm{y}$ $3,89 \%$, en salchichas adicionadas con una mezcla de piel de cerdo y fibra de trigo en diferentes concentraciones, en donde, igualmente, la de menor pérdida fue la de mayor concentración de dicha mezcla. Del mismo modo, también concuerdan con el trabajo publicado por Pintado et al. (2018), quienes fabricaron salchichas de cerdo tipo longaniza, bajas en grasa, adicionadas con fibra de chía (Salvia bispánica L.) y fibra de avena (Avena sativa L.), en donde se presentó una menor diminución en las pérdidas de agua por cocción (1,60\%). De los análisis presentados en los artículos citados, se puede concluir que, al igual que en esta investigación, la adición de diferentes tipos de fibras en matrices cárnicas mejora el rendimiento en cocción, lo cual, tiene importancia en las características de textura y, por ende, en la aceptación de los productos finales. Por otro lado, la mezcla de PCFQ afectó la emulsión de las diferentes salchichas (Tabla 3). El contenido de grasa y la pérdida de agua por cocción disminuyeron en comparación con la salchicha control. Esto se puede explicar por la mayor encapsulación de glóbulos de grasa por parte de las moléculas de proteína de los filetes de tilapia durante la emulsificación (Lee \& Chin, 2016).

Las características de textura de los productos cárnicos, se alteran, significativamente, al reducir el contenido de grasas y al aumentar el contenido de agua, ya que estos componentes influyen directamente en la estructura morfológica de los tejidos y en las interacciones de estos con los componentes adicionados en la formación de la emulsión cárnica. El perfil de textura de los productos cárnicos y pesqueros está relacionado con la capacidad de retención de agua por parte de la proteína. En la tabla 4, se muestran los resultados del TPA para las salchichas elaboradas. Al adicionar PCFQ, la dureza se vio afectada $(\mathrm{p}<0,05)$, menor para la salchicha control, mientras que la salchicha con $20 \%$ de PCFQ, obtuvo el mayor valor, datos que concuerdan con los presentados por Choe et al. (2013), quienes afirman que la mayor dureza se debe a que la gelatina de la piel de cerdo tiene una textura dura a bajas temperaturas. La mayor elasticidad se vio en las salchichas control $(\mathrm{p}<0,05)$. Por el contrario, los parámetros cohesividad, gomosidad y masticabilidad presentaron mayores valores en las muestras con adición de PCFQ. Según Westphalen et al. (2006), las propiedades de textura de los productos cárnicos cocidos están altamente relacionados con la gelificación de las proteínas miofibrilares, en consecuencia, las mejores características de textura estarían asociadas al aumento de la proteína soluble, lo que forma estructuras más sólidas al final del proceso de escaldado. Por otro lado, la gelificación de la estructura de la emulsión podría estar asociada a la presencia de fibra dietética y su capacidad de retención de agua (Agar et al. 2016).

La evaluación sensorial de las diferentes salchichas, se aprecia en la tabla 5. No se presentaron diferencias significativas en los parámet- 
ros de color, ternura y jugosidad, esta última, estrechamente ligada con el $\mathrm{pH}$, la capacidad de retención de agua y la pérdida de humedad por cocción (Choe et al. 2013). La adición de PCFQ no afectó significativamente el sabor y el sabor calentado de las salchichas comparadas con la salchicha control. La aceptabilidad general tuvo una variación no significativa, lo cual, muestra que la adición de PCFQ, como sustituto de grasas, no afectó los parámetros sensoriales de las salchichas analizadas, mostrando parámetros muy cercanos a los de la salchicha control. Estos resultados concuerdan con los aportados por Choe et al. (2013), quienes prepararon salchichas de cerdo bajas en grasa con adición de una mezcla de piel de cerdo y fibra de trigo, así como también los mostrados por Candogan \& Kolsarici (2003), quienes elaboraron salchichas de carne de res con adición de carragenano con gel de pectina.
La adición de PCFQ, como sustituto de grasas, conllevó a una buena aceptabilidad sensorial de las salchichas de tilapia bajas en grasa. El contenido de ésta, se redujo en valores que fluctuaron entre el 12,3 y el 48,2\%, en proporción directa al aumento de la concentración de PCFQ adicionada. Enconsecuencia, el valor calórico igualmente se redujo en porcentajes que variaron entre 3,92 y 32,32\%. Por el contrario, los valores de la humedad y la proteína aumentaron $(\mathrm{p}<0,05)$, con relación a la salchicha control. La mezcla de piel de cerdo, agua y fibra de quinua utilizada en la elaboración de las salchichas tuvo una muy buena retención de agua y, en las concentraciones utilizadas, dio como resultado una pérdida reducida de humedad por cocción. Con relación a las coordenadas de color, los parámetros de brillo $\left(\mathrm{L}^{*}\right)$ y la coordenada $\left(\mathrm{a}^{*}\right)$ que determina el contraste rojo-verde son los parámetros más informativos para medir el color en salchichas

Tabla 5. Efecto de la mezcla de piel de cerdo y fibra de quinua (PCFQ), en los parámetros sensoriales de las salchichas elaboradas.

\begin{tabular}{|c|c|c|c|c|c|}
\hline \multirow{2}{*}{ Parámetro } & \multicolumn{5}{|c|}{ Tratamiento (nivel de PCFQ*) } \\
\cline { 2 - 6 } & \multirow{2}{*}{ Control } & $\mathbf{T}_{\mathbf{1}}$ & $\mathbf{T}_{\mathbf{2}}$ & $\mathbf{T}_{\mathbf{3}}$ & $\mathbf{T}_{\mathbf{4}}$ \\
\cline { 2 - 6 } & & $\mathbf{8 \%}$ & $\mathbf{1 2} \%$ & $\mathbf{1 6} \%$ & $\mathbf{2 0} \%$ \\
\hline Color & $5,78 \pm 0,44$ & $6,12 \pm 0,56$ & $6,54 \pm 0,49$ & $6,55 \pm 0,62$ & $6,55 \pm 0,42$ \\
\hline Sabor & $6,18 \pm 0,34$ & $6,01 \pm 0,39$ & $6,03 \pm 0,42$ & $6,01 \pm 0,31$ & $5,83 \pm 0,54$ \\
\hline Ternura & $6,17 \pm 0,57$ & $6,06 \pm 0,62$ & $6,00 \pm 0,55$ & $5,94 \pm 0,40$ & $5,92 \pm 0,61$ \\
\hline Jugosidad & $5,62 \pm 0,23$ & $5,69 \pm 0,29$ & $5,99 \pm 0,43$ & $6,07 \pm 0,36$ & $6,10 \pm 0,51$ \\
\hline Sabor calentado & $5,91 \pm 0,55$ & $5,87 \pm 0,48$ & $5,78 \pm 0,52$ & $5,31 \pm 0,46$ & $5,03 \pm 0,39$ \\
\hline Aceptabilidad general & $5,98 \pm 0,56$ & $5,83 \pm 0,43$ & $5,80 \pm 0,48$ & $5,62 \pm 0,55$ & $5,48 \pm 0,61$ \\
\hline
\end{tabular}

Todos los valores son medios \pm desviación estándar de tres repeticiones.

*La proporción de la formulación de la mezcla de piel de cerdo y fibra de quinua fue piel de cerdo: agua: fibra de quinua = 2:2:1.

Color, sabor, ternura, jugosidad, sabor calentado y aceptabilidad general: $1=$ No me gusta $7=$ Me gusta muchísimo.

(Savadkoohi et al. 2014). Los tratamientos con PCFQ, comparados con la muestra control, presentaron valores de brillo $\left(\mathrm{L}^{*}\right)(\mathrm{p}<0,05)$ más bajos, lo cual, se atribuye a que la mezcla de piel de cerdo y harina de quinua diluida en agua tiene un tono marrón. Lo anterior concuerda con el trabajo presentado por Savadkoohi et al. (2014), quienes incorporaron bagazo de tomate $7 \%(\mathrm{p} / \mathrm{p})$ en la elaboración de salchichas, tipo Frankfurt. La coordenada $\left(a^{*}\right)$ del CIELab, no presentó diferencias significativas entre las salchichas control y las muestras elaboradas. Los valores de la coordenada ( $\left.b^{*}\right)$, que define el contraste azul-amarillo, no mostraron ningún efecto significativo $(\mathrm{p}<0,05)$, en todas las concentraciones de las salchichas analizadas. El ángulo de tonalidad (h) para las salchichas adicionadas con PCFQ, no presentaron un aumento significativo $(p<0,05)$ en comparación con la salchicha control, lo cual, se atribuye a que los valores de las coordenadas ( $\left.\mathrm{a}^{*}\right)$ (rojo-verde) y (b*) (azul-amarillo) se mantuvieron estables, situación similar que se aprecia para el índice de saturación C. En consecuencia, la adición de PCFQ en salchichas elaboradas con filetes de tilapia no afectó significativamente los parámetros de color, jugando un papel clave en la aceptación sensorial de este tipo de productos cárnicos, como lo afirman en su trabajo, desarrollado en salchichas tipo emulsión, con adición de polvo de alcachofa
(Afoakwah et al. 2015). Con relación al perfil de textura PTA, la adición de PCFQ mejoró las características de dureza, de cohesividad, de gomosidad y de masticabilidad, en proporción directa al aumento de la concentración de la misma, mientras que la elasticidad se vio disminuida, aunque no en forma significativa, debido, probablemente, a las características químicas y la composición proximal de la mezcla PCFQ, en donde, gracias a la adición de piel de cerdo, se incrementó el contenido de colágeno, proteína determinante en la elasticidad del producto final. De la presente investigación, se puede concluir que la inclusión de una mezcla de piel de cerdo, agua y fibra de quinua, de hasta un 20\% de sustitución en la formulación de salchichas tipo Frankfurt, elaboradas a partir de filetes de tilapia, puede ser considerada como una alternativa importante para la disminución de grasas saturadas de origen animal, en dichos productos. Se requieren otras investigaciones, que permitan determinar la cantidad máxima de la mezcla utilizada a reemplazar en salchichas de tilapia, sin que se afecten las propiedades nutricionales, fisicoquímicas y sensoriales de estos productos.

Conflictos de intereses: El manuscrito fue preparado y revisado con la participación de todos los autores, quienes declaramos que no 
existe ningún conflicto de intereses que ponga en riesgo la validez de los resultados presentados.

\section{REFERENCIAS}

1. AFOAKWAH, N.A.; DONG, Y.; ZHAO, Y.; XIONG, Z.; OWUSU, J.; WANG, Y.; ZHANG, J. 2015. Characterization of Jerusalem artichoke (Helianthus tuberosus L.) powder and its application in emulsion-type sausage. LWTFood Sci. Techn. 64(1):74-81. https://doi.org/10.1016/j. lwt.2015.05.030

2. AGAR, B.; GENCCELEP, H., SARICAOGLU, F.T.; TURHAN, S. 2016. Effect of sugar beet fiber concentrations on rheological properties of meat emulsions and their correlation with texture profile analysis. Food Biop. Proc. 100(Part A):118-131. https:/ /doi.org/10.1016/j.fbp.2016.06.015

3. ARZAPALO, D.; HUAMAN, K.; QUISPE, M.; ESPINOSA, C. 2015. Extracción y caracterización del almidón de tres variedades de quinua (Chenopodium quinoa Willd) negra collana, pasankalla roja y blanca junín. Rev. Soc. Quim. Perú. 81(1):44-54.

4. ASSOCIATION OF OFFICIAL ANALYTICAL CHEMISTS -AOAC-. 2003. Official methods of analysis of the Association of Official Analytical Chemists. $17^{\text {th }}$ ed. Gaithersburg: AOAC.

5. AUTORIDAD NACIONAL DE ACUICULTURA Y PESCA -AUNAP-. 2013. Diagnóstico del estado de la acuicultura sostenible en Colombia. Disponible desde Internet en: http://www.aunap.gov.co

6. BENELLI, J.; TONIAZZO, R.C.; PRESTES, R.C.; TRES, M.V. 2015. Development and utilizatión of pork skin emulsion in mortadella as a soy protein substitute. Intern. Food Res. J. 22(5):2126-2132.

7. BESSA, D.P.; TEIXEIRA, C.E.; FRANCO, R.B.; FREITAS, M.Q.; GUERRA, M.L.; CONTE, C.A.; VARON, L.V.; ALVES, F.; TEIXEIRA, E. 2016. Functional sausages made from mechanically separated tilapia meat. Italian J. Food Sci. 28(3):426-439. https://doi.org/10.14674/1120-1770/ ijfs.v165

8. CABELLO, A.M.; MARTÍNEZ, Z.; VILLEGAS, L.; FIGUERA, B.; MARCANO, L.; GÓMEZ, A.; VALLENILLA, O. 2005. Fauna acompañante del camarón como materia prima para la elaboración de productos pesqueros. Zootecnia Tropical 23(3):217-230.

9. CANDOGAN, K.; KOLSARICI, N. 2003. The effects of carrageenan and pectin on some quality characteristics of low-fat beef frankfurters. Meat Sci. 64(2):199-206. https:/ / doi.org/10.1016/S0309-1740(02)00181-X
10. CHEETANGDEE, N. 2017. Characteristics of sausages as influenced by partial replacement of pork-fat using pre-emulsified soybean oil stabilized by fish proteins isolate. Agric. Nat. Res. 51(4):310-318. https://doi.org/10.1016/j. anres.2017.04.006

11. CHOE, J.H.; KIM, H.Y.; LEE, J.M.; KIM, Y.J.; KIM, C.J. 2013. Quality of frankfurter-type sausages with added pig skin and wheat fiber mixture as fat replacers. Meat Sci. 93(4):849854. https://doi.org/10.1016/j.meatsci.2012.11.054

12. CHOI, Y.S.; CHOI, J.H.; HAN, D.J.; KIN, H.Y.; LEE, M.A.; KIM, W.; LEE, J.W.; CHUNG, H.J.; KIM, C.J. 2010. Optimization of replacing pork back fat with grape seed oil and rice bran fiber for reduced-fat meat emulsion systems. Meat Sci. 84(1):212-218. https:/ / doi.org/10.1016/j.meatsci.2009.08.048

13. CHOI, Y.S.; KIM, H.W.; HWANG, K.E.; SONG, D.H.; CHOI, J.H.; LEE, M.A.; CHUNG, H.J.; KIM, C.J. 2014. Physicochemical properties and sensory characteristics of reduced-fat frankfurters with pork back fat replaced by dietary fiber extracted from makgeolli lees. Meat Sci. 96(2 Pt A):892-900. https://doi.org/10.1016/j.meatsci.2013.08.033

14. ENSOR, S.A.; MANDIGO, R.W.; CALKINS, C.R.; QUINT, L.N. 1987. Comparative Evaluation of whey protein concentrate, soy protein isolate and calcium-reduced nonfat dry milk as binders in an emulsion-type sausage. J. Food Sci. 52(5):1155-1158. https://doi. org/10.1111/j.1365-2621.1987.tb14032.x

15. FERGUSON, L.P. 2010. Meat and cancer. Meat Sci. 84(2):308 313. https://doi.org/10.1016/j.meatsci.2009.06.032

16. FOOD AND AGRICULTURE ORGANIZATION OF THE UNITED NATIONS -FAO-. 2015a. Colombia, pesca en cifras 2014. Disponible desde Internet en: http:/ / www.fao. org/publications

17. FOOD AND AGRICULTURE ORGANIZATION OF THE UNITED NATIONS -FAO-. 2015b. Fisheries and aquaculture topics. Seafood products. Disponible desde Internet en: http://www.fao.org/fishery/topic/12253/en

18. GRANADOS-CONDE, C.; GUZMÁN-CARRILLO, L.E.; ACEVEDO-CORREA, D. 2013. Evaluación de salchichas elaboradas con carne roja de atún. Orinoquia 17(2):197201.

19. HAJKOWICZ, S.; COOK, H.; LITTLEBOY, A. 2012. Our future world: global megatrends that will change the way we live, The 12 revision. Disponible desde Internet en: http://www.csiro.au/en/Do-business/Futures/reports / our-Future-World 
20. HEMUNG, B.O.; SRIUTTHA, M. 2014. Effects of tilapia bone calcium on qualities of tilapia sausage. Kasetsart J. Nat. Sci. 48(5):790-798.

21. HLEAP, J.I.; RODRÍGUEZ, G. 2018. Physicochemical analysis of frankfurter Type sausages made with red tilapia fillet waste (Oreochromis sp.) and quinoa flour (Chenopodium quinoa W.). Braz. J. Food Techn. 21:1-8. https://doi. org/10.1590/1981-6723.10316

22. HLEAP, J.I.; VELASCO, V.A. 2010. Análisis de las propiedades de textura durante el almacenamiento de salchiichas elaboradas a partir de tilápia roja (Oreochromis sp.). Rev. Bio. Agro. $8(2): 46-56$.

23. HSU, S.Y.; SUN, L.Y. 2006. Comparisons on 10 non-meat protein fat substitutes for low-fat Kung-wans. J. Food Eng. 74(1):47-53. https://doi.org/10.1016/j.jfoodeng.2005.02.022

24. INTARASIRISAWAT, R.S.; BENJAKUL, S.; VISESSANGUAN, W.; WU, J. 2014. Effects of skipjack roe protein hydrolysate on properties and oxidative stability of fish emulsion sausages. LWT- Food Sci. Techn. 58(1):280-286. https://doi.org/10.1016/j.lwt.2014.02.036

25. LEE, C.H.; CHIN, K.B. 2016. Effects of pork gelatin levels on the physicochemical and textural properties of model sausages at different fat levels. LWT-Food Sci. Techn. 74:325-330. https://doi.org/10.1016/j.lwt.2016.07.032

26. LIMBERGER, V.M.; BRUM, F.B.; PATIAS, L.D.; DANIEL, A.P.; COMARELA, C.G.; EMANUELLI, T.; SILVA L.P. 2011. Modified broken rice starch as fat substitute in sausages. Ciênc. Tecnol. Alim. 31(3):789-792. https://doi. org/10.1590/S0101-20612011000300037

27. McAFEE, A.J.; McSORLEY E.M.; CUSKELLY, G.J.; MOSS, V.W.; WALLACE, J.M.; BONHAM, M.P.; FEAROM, A.M. 2010. Red meat consumption: An overview of the risks and benefits. Meat Sci. 84(1):1-13. https://doi.org/10.1016/j. meatsci.2009.08.029

28. MIRANDA, M.; VEGA-GÁLVEZ, A.; QUISPE-FUENTES, I.; RODRÍGUEZ, M.J.; MAUREIRA, H.; MARTÍNEZ, E.A. 2012. Nutritional aspects of six quinoa (Chenopodium quinoa Willd) ecotypes from three geographical areas of Chile. Chilean J. Agric. Res. 72(2):175-181. https://doi. org/10.4067/S0718-58392012000200002

29. OSBURN, W.N.; MANDIGO, R.W.; ESKRIDGE, K.M. 1997. Pork skin connective tissue gel utilization in reduced-fat bologna. J. Food Sci. 62(6):1176-1182. https:/ / doi.org/10.1111/j.1365-2621.1997.tb12239.x
30. PACHECO-PÉREZ, W.A.; RESTREPO-MOLINA, D.A.; SEPÚLVEDA- VALENCIA, J.U. 2011. Revisión: uso de ingredientes no cárnicos como reemplazantes de grasa en derivados cárnicos. Rev. Fac. Nac. Agron. 64(2):6257-6264.

31. PAL, G.K.; SURESH, P.V. 2016. Sustainable valorization of seafood by-products: Recovery of collagen and development of collagen-based novel functional food ingredients. Innov. Food Sci. Emerg. Techn. 37(Part B):201-215. https://doi. org/10.1016/j.ifset.2016.03.015

32. PINTADO, T.; HERRERO, A.M.; JIMÉNEZ-COLMENERO, F.; PASQUALIN-CAVALHEIRO, C.; RUIZ-CAPILLAS, C. 2018. Chia and oat emulsion gels as new animal fat replacers and healthy bioactive sources in fresh sausages formulation. Meat Sci. 135:6-13. https://doi.org/10.1016/j. meatsci.2017.08.004

33. PINZÓN-ZÁRATE, L.X.; HLEAP-ZAPATA, J.I.; ORDÓÑEZ-SANTOS, L.E. 2015. Análisis de los parámetros de color en salchichas Frankfurt adicionadas con extracto oleoso de residuos de chontaduro (Bactris gasipaes). Inf. Tecnol. 26(5):45-54. https://doi.org/10.4067/S071807642015000500007

34. REDDY, V.K.; RAJU, C.V.; SHAFIK, U.; CHANDRA, M.V.; LAKSHMISHA, I.P. 2020. Influence of protein isolates from Pangas processing waste on physico-chemical, textural, rheological and sensory quality characteristics of fish sausages. LWT 117:108662. https://doi.org/10.1016/j. lwt.2019.108662

35. RUEDA-LUGO, U.; GONZÁLEZ-TENORIO, R.; TOTOSAUS, A. 2006. Sustitución de lardo por grasa vegetal en salchichas: incorporación de pasta de aguacate. Efecto de la inhibición del oscurecimiento enzimático sobre el color. Ciênc. Tecnol. Aliment. 26(2):441-445. https://doi. org/10.1590/S0101-20612006000200030

36. SAVADKOOHI, S.; HOOGENKAMP, H.; SHAMSI, K.; FARAHNAKY, A. 2014. Color, sensory and textural attributes of beef frankfurter, beef ham and meat-free sausage containing tomato pomace. Meat Sci. 97(4): 410-418. https:/ / doi.org/10.19026/ajfst.16.5962

37. SERIN, S.; SAYAR, S. 2017. The effect of the replacement of fat with carbohydrate-based fat replacer on the dough properties and quality of the baked pogaka: a traditional high-fat bakery product. Food Sci. Techn. 37(1):25-32. https://doi.org/ 10.1590/1678-457x.05516

38. SHAHIDI, F.; AMBIGAIPALAN, P. 2015. Novel functional food ingredients from marine sources. Cur. Opin. Food Sci. 2:123-129. 
39. SORLAND, G.H.; LARSEN, P.M.; LUNDBY, F.; RUDI, A.P.; GUIHENEUF, T. 2004. Determination of total fat and moisture content in meat using low field NMR. Meat Sci. 66(3):543-550. https://doi.org/10.1016/S0309$1740(03) 00157-8$

40. SOUZA, S.C.; FRAGOSO, S.P.; PENNA, C.R.A.; ARCANJO, N.M.O.; SILVA, F.A.P.; FERREIRA, V.C.S.; BARRETO, M.D.; ARAÚJO, I.B. 2017. Quality parameters of frankfurter-type sausages with partial replacement of fat hydrolyzed collagen. LWT-Food Sci. Techn. 76(Part B):320-325. https://doi.org/10.1016/j.lwt.2016.06.034

41. TOKUSOGLU, O.; KEMAL, U. 2003. Fat replacers in meat products. Pakistan J. Nutrit. 2(3):196-203.

42. WESTON, A.R.; ROGERS, R.W.; ALTHEN, T.G. 2002. The role collagen in meat tenderness. The Profes. Anim. Scient. 18(2):107-111. https://doi.org/10.15232/S10807446(15)31497-2

43. WESTPHALEN, A.D.; BRIGGS, J.L.; LONERGAN, S.S.M. 2006. Influence of muscle type on rheological properties of porcine myofibrillar protein during heat-induced gelation. Meat Sci. 72(4):697-703. https://doi.org/10.1016/j. meatsci.2005.09.021

44. YANG, H.; SHU, Z. 2014. The extraction of collagen protein from pigskin. J. Chem. Pharm. Res. 62(2):683-687.

45. YILMAZ, I. 2005. Physicochemical and sensory characteristics of low-fat meatballs with added wheat bran. J. Food Eng. 69(3):369-373. https://doi.org/10.1016/j.jfoodeng.2004.08.028

46. ZAKARIA, N.A.; SARBON, N.M. 2018. Physicochemical properties and oxidative stability of Fish emulsion sausages as influenced by snakehead (Channa striata) protein hydrolysate. LWT-Food Sci. Techn. 94:13-19. https://doi. org/10.1016/j.lwt.2018.04.026

47. ZHAO, Y.; HOW, Q.; ZHUANG, X.; WANG, Y.; ZHOU, G.; ZHANG, W. 2018. Effect of regenerated cellulose fiber on the physicochemical properties and sensory characteristics of fat-reduced emulsified sausage. LWT-Food Sci. Techn. 97:157-163. https://doi.org/10.1016/j.lwt.2018.06.053 